\title{
The influence of buffer time distributions in delay propagation modelling of railway networks
}

\author{
Stephan Zieger ${ }^{\mathrm{a}, *}$, Norman Weik $^{\mathrm{a}}$, Nils Nießen ${ }^{\mathrm{a}}$ \\ ${ }^{a}$ Institute of Transport Science, RWTH Aachen University, 52074 Aachen
}

\begin{abstract}
Buffer times are an important factor in railway timetable design preventing the propagation of delays and ensuring timetable robustness. Determining the required amount of buffer times, such that a certain level of service quality is achieved, falls within the responsibility of railway capacity analysis. This is why capacity analysis is intrinsically linked to delay propagation modelling. Currently, delay propagation modelling in this context relies on the assumption of random, exponentially distributed or deterministic buffer times. Real-world timetables tend to deviate from this behaviour, such that a more general modelling of buffer time distributions is desirable. In this paper the impact of different buffer time distributions on the build-up of knock-on delays in delay propagation modelling is analysed using a Monte-Carlo simulation approach. It is shown that the choice of distribution has a significant impact on performance metrics. In a sensitivity analysis line capacity is observed to vary by as much as $17 \%$ as a function of the underlying buffer time statistics in the investigated scenarios.
\end{abstract}

Keywords: buffer times, delays, stochastic models, capacity, railways, long term planning

\section{Introduction and Literature Review}

Buffer times correspond to an increase of headway times beyond minimum separation times, which are enforced by track occupation constraints in interlocking. Consequently, they impede the formation of train conflicts and counteract the transmission of delays between trains (knock-on delays) resulting from small primary delays. These knock-on delays appear when a delayed train hinders other trains by still occupying parts of the scheduled route and therefore thwarting other trains from passing or crossing (Yuan and Hansen, 2007).

Depending on their location and size, the effectiveness of buffer times varies and timetables exhibit different degrees of robustness against disruptions. Ensuring and optimising timetable robustness by effectively allocating buffer times is a delicate task that has attracted a lot of interest both in academia and industry

\footnotetext{
* This is the Authors' Accepted Manuscript of the following article: S. Zieger, N. Weik, N. Nießen, The influence of buffer time distributions in delay propagation modelling of railway networks, Journal of Rail Transport Planning \& Management 8(3-4), pp. 220-232, 2018, which has been published in final form at https://doi.org/10.1016/j.jrtpm.2018.09.001. (C) 2018. This manuscript version is made available under the CC-BY-NC-ND 4.0 license http://creativecommons.org/ licenses/by-nc-nd/4.0/

* Corresponding author Email address: zieger@via.rwth-aachen.de (Stephan Zieger) URL: http://www.via.rwth-aachen.de/english/start.php ()
} 
(see Lusby et al. (2017) for a recent survey of robustness in railway planning). While many methods in the field rely on (iterative) a-posteriori simulation of disturbed timetables, robust optimisation approaches have also found widespread application in recent years.

Salido et al. (2008) discuss different robustness metrics and present analytic approaches to compare the robustness of timetables from a railway operator's point of view based on disruption scenario simulations. A comparable methodology adopting a passenger centred approach has been proposed by Takeuchi et al. (2007). Here, robustness is assessed in terms of passenger disutility. The analysis also relies on Monte Carlo simulation of operations in view of disruptions. A more elaborate delay propagation simulation tool and its use in robustness analysis of timetables are discussed by Corman et al. (2014): The authors set up a multi-level dispatching scheme, where regional rescheduling is coordinated on network scale. Normal and disruption timetables are analysed with respect to train delays and passenger travel times.

Cacchiani and Toth (2012) provide an overview of robust optimisation approaches to the (capacitated) train timetabling problem. Both cyclic and non-cyclic timetabling approaches are discussed. More recently, a knapsack approach for the buffer time allocation problem has been proposed by Jovanović et al. (2017). A robust joint routing and scheduling model for densely used station areas is discussed by Dewilde et al. (2014). The approach has been tested for the Brussels Central area, where a decrease of delay propagation of $25 \%$ with respect to previous timetables is found.

In Khoshniyat and Peterson (2017), a buffer time optimisation framework is studied where buffer times are allocated in relation to the headway times between trains. This is motivated by the fact that long train running sections exhibit larger degrees of uncertainty in operations. A new notion of robustness - robustness in critical points - has been introduced by Andersson et al. (2013). The criticality of timetable reserves with respect to the train sequence in timetables is analysed. An evaluation of the effectiveness of a purely analytic ex-ante timetable optimisation approach based on the robustness critical points has been provided in Solinen et al. (2017) using microscopic train simulations.

In the scheduling context, robustness analysis is predominantly concerned with analysing and optimising timetables where the number of trains is taken to be fix. The feasibility of the operational concept is generally assumed to have been ensured by capacity analysis beforehand. Only very few publications consider the possibility to cancel train paths (Cacchiani et al., 2016) or to schedule additional train paths within a given timetable (Burdett and Kozan, 2009; Khoshniyat and Törnquist Krasemann, 2017). In Khoshniyat and Törnquist Krasemann (2017) a MILP approach for scheduling additional train paths or maintenance works with minimal timetable deviations is investigated. The method allows to deduce a-posteriori robustness parameters in scheduling.

Capacity planning, by contrast, aims to determine the number of trains which can be operated, such that a pre-defined level of service is maintained (UIC, 2004). This level of service is defined in terms of quality indicators such as train punctuality or amount of delays (Abril et al., 2008). As a consequence, capacity analysis methods necessarily involve some kind of delay propagation modelling. Even techniques relying on metrics like infrastructure occupation, such as the widespread UIC schedule compression method (UIC, 2013), require standards for the level of service which are calibrated either based on expert knowledge or delay modelling (Wendler et al., 2002).

In delay modelling for capacity analysis, two main streams can be distinguished: Timetable-centred 
approaches, which investigate train delays forming in rescheduling a perturbed, fully constructed timetable. These approaches are closely linked to the previously discussed approaches in timetable stability and robustness analysis. Their main area of use is tactical planning, when timetable structure and train sequence are already largely known (Delorme et al., 2009; Adenso-Diaz et al., 1999). The second stream consists of probabilistic approaches, which do not require a fully constructed timetable. Train interactions and conflicts are modelled in terms of queueing systems (Huisman et al., 2002; Wendler, 2007; Weik and Nießen, 2017) or stochastic models based on probability distribution functions describing train arrival/departure times (Schwanhäußer, 1974; Weik et al., 2016), running times (Meester and Muns, 2007) or delays (Yuan, 2006; Büker and Seybold, 2012). A linear programming approach for complex route nodes, where conflict probabilities between train paths are input for a delay minimisation problem is discussed by Mussone and Calvo (2013). A similar train path exclusion technique is used for route nodes to obtain simplified queueing descriptions with aggregated service centers in Nießen (2008).

The focus of the present paper is on the second stream of probabilistic approaches for delay propagation modelling in capacity analysis. In this context, the representation and analysis of suitable delay distribution functions has attracted a lot of interest (see, e.g., Yuan (2006); Wen et al. (2017)). By contrast, the assumptions made on buffer times, which are equally crucial for delay build-up, have received much less attention. Existing approaches assume buffer times to be either deterministic (Büker and Seybold, 2012; Yuan, 2006) or exponentially distributed (Schwanhäußer, 1974; Weik et al., 2016). In Nie and Hansen (2005), however, it has been shown that real-world data for timetable buffer times on railway lines are not necessarily exponentially distributed. A more detailed analysis of buffer time statistics is missing, so far.

This paper aims to close this gap by providing a rigorous analysis of the influence of buffer time distributions on the formation of knock-on delays. The analysis is based on a stochastic event-based simulation, which allows to analyse the build-up of knock-on delays from primary delays, locally. Note that this does not mean that the results are limited to local delay propagation models like the ones discussed by Weik et al. (2016). They equally transfer to network models, such as (Büker and Seybold, 2012; Yuan, 2006), which are composed of individual events, where buffer times enter in the convolution integrals describing delay propagation. For comparison the simulation is validated against the STRELE method (Schwanhäußer, 1994), an analytic method which forms the standard approach used by German infrastructure manager DB Netz AG for delay prognosis and capacity assessment of railway lines (DB Netz AG, 2008).

A more detailed discussion of the STRELE framework as well as the event-based simulation approach applied in this work is given in the following section. Section 3 - which is the main part of this paper contains the investigation of the relation between buffer time distributions and knock-on delays. Apart from the analysis of how these distributions bear on knock-on delays, we also provide a brief discussion of the computation complexity of the approach as well as a sensitivity analysis.

\section{Method}

Subsequently, the methodology used in this work is presented. We start by introducing the STRELE framework, which is the standard method for the assessment of knock-on delays in Germany (DB Netz AG, 2008). The second part describes the structure of the simulation tool and gives an idea of the effects of 
synchronous and asynchronous conflict resolution techniques in simulation. In conclusion of the section a short summary of the required input data for STRELE and simulation is given.

\subsection{STRELE}

The STRELE approach (Schwanhäußer, 1974) to compute knock-on delays is based on a decomposition of railway lines into independent directional tracks. Using a probabilistic propagation technique for train interactions as well as results from queueing theory an approximation of the mean knock-on delays of trains is obtained. The method is commonly used in Germany to determine railway capacity and has been implemented in various software tools, including LUKS $^{\circledR}$ ) and SLS (Janecek and Weymann, 2010; Schwanhäußer et al., 2000). While conceptualised for double-track railway lines the STRELE-formula can be used for single-track railway lines with minor modifications, as well.

The STRELE formula, which is at the center of the approach, allows to determine the expected knock-on delay $t$ for a railway line segment between two overtaking stations. It is given by

$$
\begin{aligned}
& t=\left(p_{d e l}-\frac{p_{d e l}^{2}}{2}\right) \cdot \frac{\bar{t}_{d e l}^{2}}{\bar{t}_{b}+\bar{t}_{d e l} \cdot\left(1-\exp \left(-\frac{\bar{h}}{t_{d e l}}\right)\right)} \\
& \cdot\left[p_{e q}\left(1-\exp \left(-\frac{\bar{h}_{e q}}{\bar{t}_{d e l}}\right)\right)^{2}+\left(1-p_{e q}\right) \frac{\bar{h}_{d i f f}}{\bar{t}_{d e l}}\left(1-\exp \left(-\frac{2 \bar{h}_{d i f f}}{\bar{t}_{d e l}}\right)\right)+\frac{\bar{h}}{\bar{t}_{b}}\left(1-\exp \left(-\frac{\bar{h}}{\bar{t}_{d e l}}\right)\right)\right]
\end{aligned}
$$

with

$p_{\text {del }}-$ probability of primary delay,

$\bar{t}_{d e l}$ - average delay of the delayed trains,

$\bar{t}_{b}$ - average buffer time,

$p_{e q}-$ probability of trains with equal rank,

$\bar{h}$ - average minimum headway time,

$\bar{h}_{e q}$ - average minimum headway time between trains with equal rank,

$\bar{h}_{\text {diff }}$ - average minimum headway time between trains with different rank.

The computation of knock-on delays follows the principle of calculating the first-order delays and upscaling the result by a factor which incorporates a heavy traffic regime and queueing system results to estimate the delays of higher order.

To assess the quality in operation, a level of service has to be defined which regulates the maximum admissible knock-on delays in railway operations. The standard for quality in Germany is defined in Directive 405 of DB Netz AG (DB Netz AG, 2008). A more extensive and rigorous discussion of the STRELEframework and its use in capacity analysis can be found in Weik et al. (2016).

\subsection{Simulation Environment}

For the simulation, an event-based Monte-Carlo approach is applied. Knock-on delays are calculated in a three-step procedure. A visualisation of the procedure is given in Algorithm 1 (c.f. Appendix A). 


\subsubsection{Schedule Generation}

By setting a number of trains $n$ with $c$ classes, i.e. sets of trains with similar driving characteristics, a schedule is generated from a random sequence of trains. As both directional tracks are operated independently and no overtakings are possible, blocking time stairways of a segment can be merged into one block.

Hence, the first train starts at time $t_{\text {sched }, 1}=0$ followed by a second train at time $t_{\text {sched }, 2}=t_{\text {sched }, 1}+$ $h_{1,2}+b_{1,2}$ with minimum headway time $h_{1,2}$, which is a technical constraint. Additionally, buffer time $b_{1,2}$ is added, which aims to ensure adequate quality in operations by minimising the transmission of primary delays, e.g. due to bad weather, signal or train malfunction or high passenger load. The start times $t_{\text {sched,i }}$ of the following trains are calculated in the same manner:

$$
t_{\text {sched }, i}=t_{\text {sched }, i-1}+h_{i-1, i}+b_{i-1, i} \text { for train } i \in\{2, n\} .
$$

Note that minimum headway times are fully determined by train type and infrastructure and the amount of buffer times in the schedule lies within the responsibility of the infrastructure manager (timetable construction process). In the simulation framework the effects of different buffer time distributions can be tested in case the schedule is not known. On the other hand, given a schedule, a buffer time distribution can be fitted and knock-on delays can be calculated and evaluated.

\subsubsection{Delay Implementation}

Infrastructure managers have empirical train specific values of delay probability and average delay time within their network. The data is either based on the values mentioned in the rulebook (in Germany, for instance, in DB Netz AG (2008)) or based on the (approximate) evaluation of real world delay statistics of railway lines. However, in the latter case the separation of primary and knock-on delays is an important issue, for which no fully satisfactory solution has been found so far. Several locally successful attempts to distinguish primary and knock-on delays have been made, e.g. by real world data analysis and mining techniques in Daamen et al. (2009), Goverde and Meng (2011) and Cule et al. (2011).

In the simulation environment, the trains $i=1, \ldots, n$ are affected by primary delay with probability $p_{d e l, i}$ and are delayed by $t_{d e l, i}$ on average in that case. At the end of the delay implementation the schedule usually contains conflicts, which have to be resolved.

\subsubsection{Rescheduling}

Resolving the induced conflicts can be basically handled in two different manners - regarding priorities in service (asynchronous) or resolving the conflicts in ascending order in time without inclusion of ranks (synchronous). In asynchronous simulation, trains are scheduled ascending in ranks, whereas in synchronous simulation trains are treated as equally ranked. The first has the advantage of giving precedence for trains which are more important for the infrastructure manager, e.g. long distance trains prior to local trains and freight trains, while the latter is more efficient in terms of network capacity. In case of high traffic load the method of operation is mostly First-Come-First-Served, but in slightly or medium perturbed operations priorities should persist. In this paper, we examine results based on asynchronous rescheduling to achieve comparable results with the analytical calculation (STRELE formula, c.f. Section 2.1). The simulation however is highly flexible and any rescheduling method can be used in general. 


\subsubsection{Evaluation of a Simulation Run}

To obtain knock-on delays $t_{\text {knock-on, } i}$ of a train $i$ the planned start time $t_{\text {sched, } i}$ and the primary delay $t_{d e l, i}$ (that may be 0 as well) are subtracted from the actual start time $t_{a c t, i}$ :

$$
t_{\text {knock-on, }, i}=t_{\text {act }, i}-\left(t_{\text {sched }, i}+t_{\text {del }, i}\right)
$$

The actual starting time is calculated based on the rescheduling policy and reflects the shift due to train path conflicts. For notational simplicity we define the operational starting time $t_{o p, i}=t_{s c h e d, i}+t_{d e l, i}$ of a train as its earliest starting time after the primary delays are inserted.

Assume that during the rescheduling process all trains (at least 2) are always in chronological order and are sorted according to Section 2.2.3 with respect to their rank. The first train in the list can be scheduled without restrictions. The second train can only have a conflict with the first train. Hence, $t_{a c t, 2}=\max \left(t_{o p}, t_{a c t, 1}+h_{1,2}\right)$.

All the following trains $i$ are rescheduled depending on their position in the regime of operation:

(a) If the train can be scheduled in front of the first train, i.e. $t_{o p, i}+h_{i, 1}<t_{a c t, 1}$, set $t_{a c t, i}=t_{o p, i}$. Otherwise, proceed to (b).

(b) If the train can be scheduled between the other rescheduled trains, the following two conditions have to be checked. If one is true, the train is scheduled as described. Otherwise, repeat the procedure for all the rescheduled trains $j$ until the end of the schedule is reached. In the case the train cannot be scheduled in the middle, proceed to (c).

(i) If the condition $\left(t_{a c t, j}+h_{j, i}+h_{i, j+1}<t_{a c t, j+1}\right) \&\left(t_{o p, i}+h_{i, j+1}<t_{a c t, j+1}\right) \&\left(t_{o p, i}<t_{a c t, j}+h_{j, i}\right)$ holds, set $t_{a c t, i}=t_{a c t, j}+h_{j, i}$

(ii) If the condition $\left(t_{a c t, j}+h_{j, i}+h_{i, j+1}<t_{a c t, j+1}\right) \&\left(t_{o p, i}+h_{i, j+1}<t_{a c t, j+1}\right) \&\left(t_{o p, i}>t_{a c t, j}+h_{j, i}\right)$ holds, set $t_{a c t, i}=t_{o p, i}$

(c) If the train can be scheduled after all other rescheduled trains, i.e. $t_{o p, i}>t_{a c t, e n d}$, set $t_{a c t, i}=$ $\max \left\{t_{o p, i}, t_{\text {act }, \text { end }}+h_{\text {end }, i}\right\}$

Depending on the evaluation the knock-on delays for a train class or for all trains are aggregated by averaging over them.

\subsubsection{Limitations of the Simulation Environment}

The simulation is designed to map the behaviour of a queuing model describing a railway segment. The segment reaches from one station providing the possibility of overtaking to the following such that for a railway line usually more than one segment needs to be considered. All segments are calculated individually and the nominal capacity in the bottleneck-segment determines the capacity of the whole railway line.

For conformity with the STRELE approach, extended running times are not considered in the simulation environment. This simplification is necessary to achieve comparability to the STRELE-framework. As the method is designed to calculate the capacity of railway lines interactions of trains within stations are not considered in the simulation. 
Extended running times could theoretically be covered in the model by introducing uncertainty in headways by allowing for variation in blocking times, for example. In order to be applicable for larger line sections spanning multiple overtaking sections the STRELE framework discussed in this model can be coupled with a gap acceptance model like the one discussed in Gast (1986), which accounts for additional delays from overtakings.

The STRELE framework is based on a simplified picture of railway operations. Still, the basic statistical principles investigated in this paper are thought to be valid for more complex models such as the OnTime framework (Büker and Seybold, 2012) or the delay propagation model proposed by Yuan (2006), as well, as these models rely on the same governing principles.

\subsection{Comparison of Input Data in STRELE and Simulation}

A brief overview over the similarities and dissimilarities of STRELE and simulation is given in Table 1. In the STRELE formula many train attributes are lost due to averaging procedures in its derivation (cf. Weik et al. (2016)). On the other hand, it provides a quick and easy to use tool to assess knock-on delays and does not suffer computational time restrictions like the simulation.

\begin{tabular}{|c|c|c|}
\hline & STRELE & Simulation \\
\hline buffer time distribution & Exponential Distribution & arbitrary \\
\hline delay distribution & mix of Exponential and Degenerate Dist. & arbitrary \\
\hline train composition & relevant & relevant \\
\hline minimum headway times & average with small variation & class specific \\
\hline priorities & arbitrary & arbitrary \\
\hline number of trains in schedule & irrelevant & relevant \\
\hline
\end{tabular}

Table 1: Comparison of input data of STRELE and simulation 


\section{Results}

We subsequently present the results of the analysis of buffer time distributions. We start by validating our simulation approach by comparison to the STRELE results. A short discussion of the computational complexity of STRELE and simulation is attached, followed by a demonstration of the main results of this paper - the examination of the influence of buffer time distributions in railway operations.

In the following discussion, the minimum headway time is set to 4 minutes, delay probability to 0.5 and train delay to 5 minutes for each train. We choose 6 model trains such that two train types per class (long-distance train, local train, freight train) are represented. Train types are drawn with equal probability and 400 trains are simulated 500 times for each data point. Unless stated otherwise, the remainder of this chapter is based on the above input.

\subsection{Validation of the Simulation for Exponentially Distributed Buffer Times}

Before analysing the impact of buffer time distributions using the simulation approach, the latter is validated against the STRELE formula. To this end, exponentially distributed buffer times are used, as the analytic STRELE framework relies on this assumption.

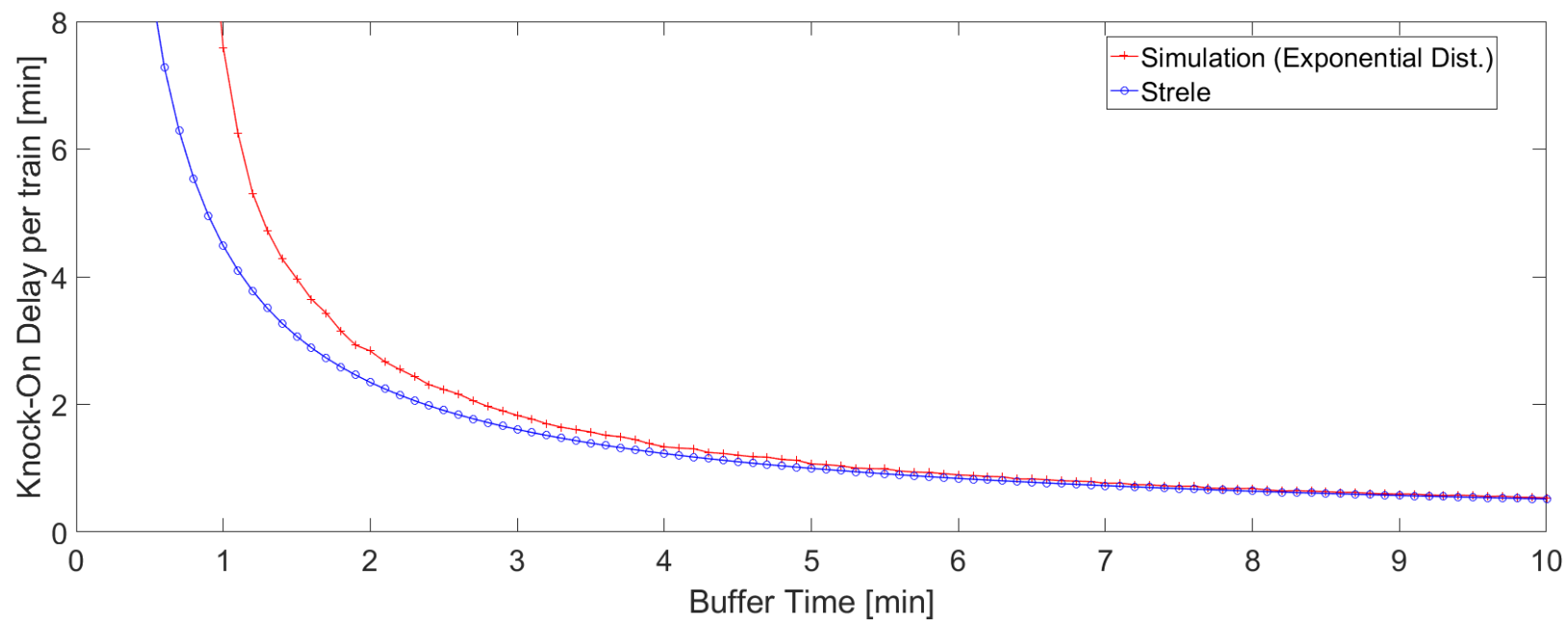

Figure 1: Comparison of knock-on delays from simulation and STRELE

In Figure 1, the knock-on delay per train in relation to the corresponding buffer time for simulation and STRELE formula is depicted. It can be seen that for average buffer times starting from 3 minutes, the results of the simulation converge reasonably fast towards STRELE. In case of small buffer times the number of classes chosen is a main factor for the amount of the expected waiting times since the probability of shifting a train with lower priority from the beginning to the end of schedule is significantly higher. When larger buffer times are realised, the trains are not packed as tightly anymore and the schedule offers more possibilities to insert lower ranked trains in earlier positions. In other test cases a similar behaviour has been observed. 


\subsection{Computational Complexity}

The implementation of the simulation environment lies within $\mathcal{O}\left(n^{2} \cdot \log (n) \cdot s \cdot t\right)$ with $n$ being the number of trains, $s$ the number of runs per point in time $t$. For example, in case of $n=400, s=500$ and $t=70$ the completion of computation takes roughly $300 \mathrm{~s}$ on an i5-6500 CPU with $3.2 \mathrm{GHz}$. With more advanced implementation techniques it might be possible to reduce the computation time by another dimension of $n$.

The calculation of STRELE is theoretically done within $\mathcal{O}\left(c^{2}\right)$, with $c$ classes/ranks, due to the fact that the preprocessing for the input data of STRELE has to evaluate the matrix of minimum headway times and other train corresponding data. In practice the calculation does not exceed one second unless having a very large number of classes.

\subsection{Determination of the Underlying Buffer Time Distribution}

In this section we discuss how the buffer time distribution for a railway line is determined: Buffer times are extracted from the actual schedule which is, in this case, performed using the construction module within

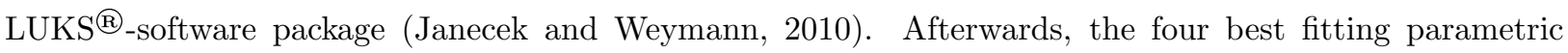
distributions are determined according to Akaike's Information Criterion (AIC) (Akaike, 1974; Kingdom and Prins, 2016) using the method of (Sheppard, 2012) to evaluate the extracted data. Therefore, the AIC is determined for a wide class of parametric distributions and then sorted in ascending order. This ordering does not give an absolute goodness-of-fit indicator, but a relative ordering of the selected distributions.

In general, for every observed distribution the absolute goodness-of-fit can be determined. In our paper, we use the Kolmogorov-Smirnov-Test (Corder and Foreman, 2014). For every distribution the so-called $p$-value is calculated. If the $p$-value is bigger than the pre-defined significance level $\alpha$ the hypothesis that the chosen distribution describes the data set cannot be rejected. In the analysis $\alpha=0.05$ is chosen as standard significance level.

\begin{tabular}{|l|l|l|l|l|l|}
\hline \multirow{4}{*}{ Fig. 2} & Distribution & Gen. Pareto & Erlang & Weibull & Gamma \\
\cline { 2 - 6 } & $\Delta$ AIC & 0 & 1.45 & 2.14 & 2.39 \\
\cline { 2 - 6 } & $p$-value & 0.234 & 0.131 & 0.188 & 0.154 \\
\cline { 2 - 6 } & Hypothesis & not rejected & not rejected & not rejected & not rejected \\
\hline \multirow{4}{*}{ Fig. 3} & Distribution & Inverse Gaussian & Gen. Pareto & Lognormal & Birnbaumsaunders \\
\cline { 2 - 6 } & $\Delta$ AIC & 0 & 1.25 & 1.86 & 1.96 \\
\cline { 2 - 6 } & $p$-value & 0.042 & 0.236 & 0.224 & 0.194 \\
\cline { 2 - 6 } & Hypothesis & rejected & not rejected & not rejected & not rejected \\
\hline \multirow{4}{*}{ Fig. 4} & Distribution & Tlocationscale & Logistic & Normal & Gen. Extreme Value \\
\cline { 2 - 6 } & $\Delta$ AIC & 0 & 1298.7 & 1303.9 & 1304.7 \\
\cline { 2 - 6 } & $p$-value & $1 \mathrm{e}-8$ & $2 \mathrm{e}-7$ & $6 \mathrm{e}-7$ & $7 \mathrm{e}-8$ \\
\cline { 2 - 6 } & Hypothesis & rejected & rejected & rejected & rejected \\
\hline
\end{tabular}

Table 2: Overview of the results depicted in Figures 2-4

In Figure 2, 3 and 4 three examples of buffer time distribution matching to real-world timetable data for different railway lines are given. For all cases the recorded actual buffer times (Data) and the fitted 


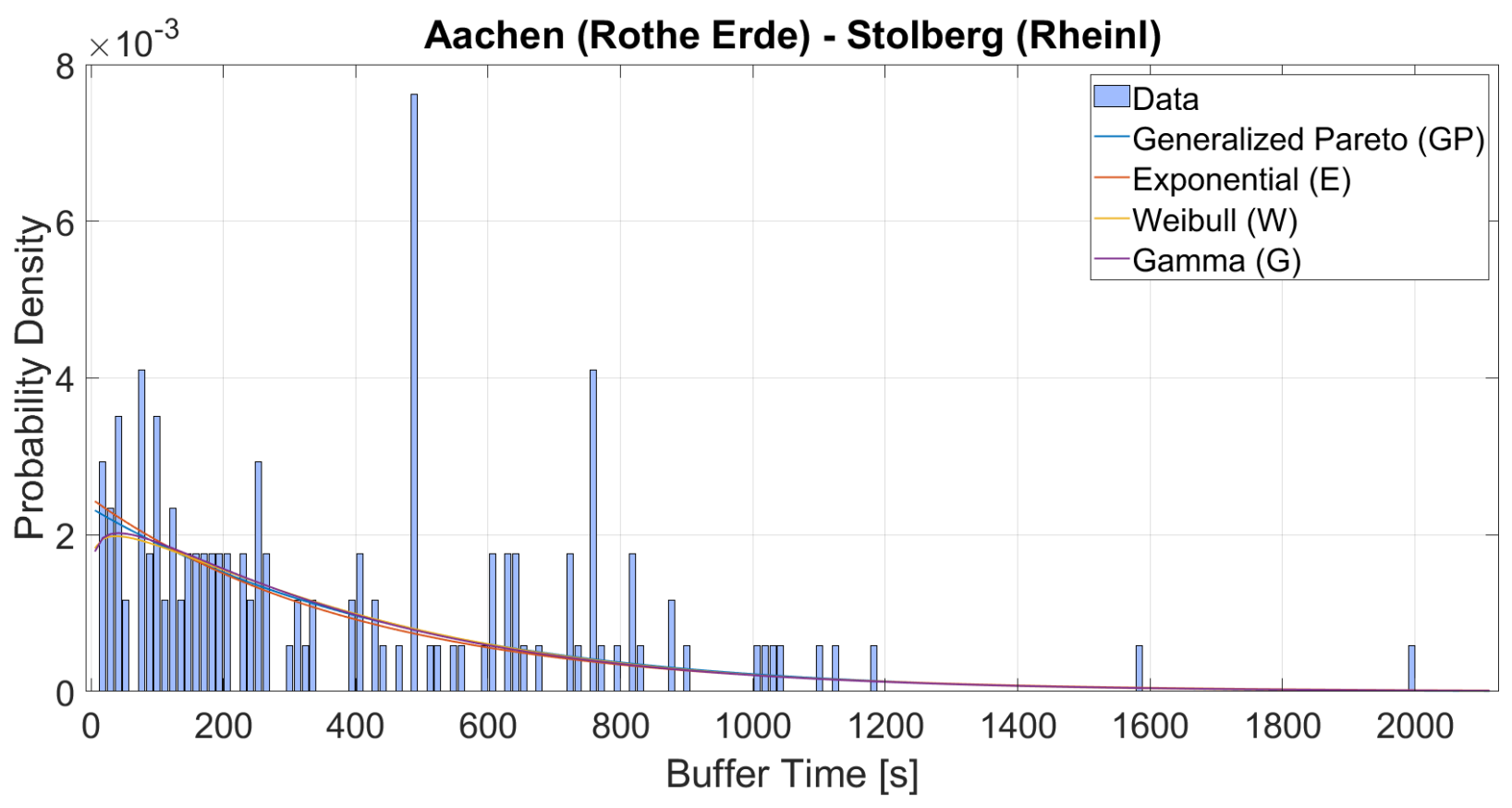

Figure 2: Probability density functions of best fitting buffer time distributions

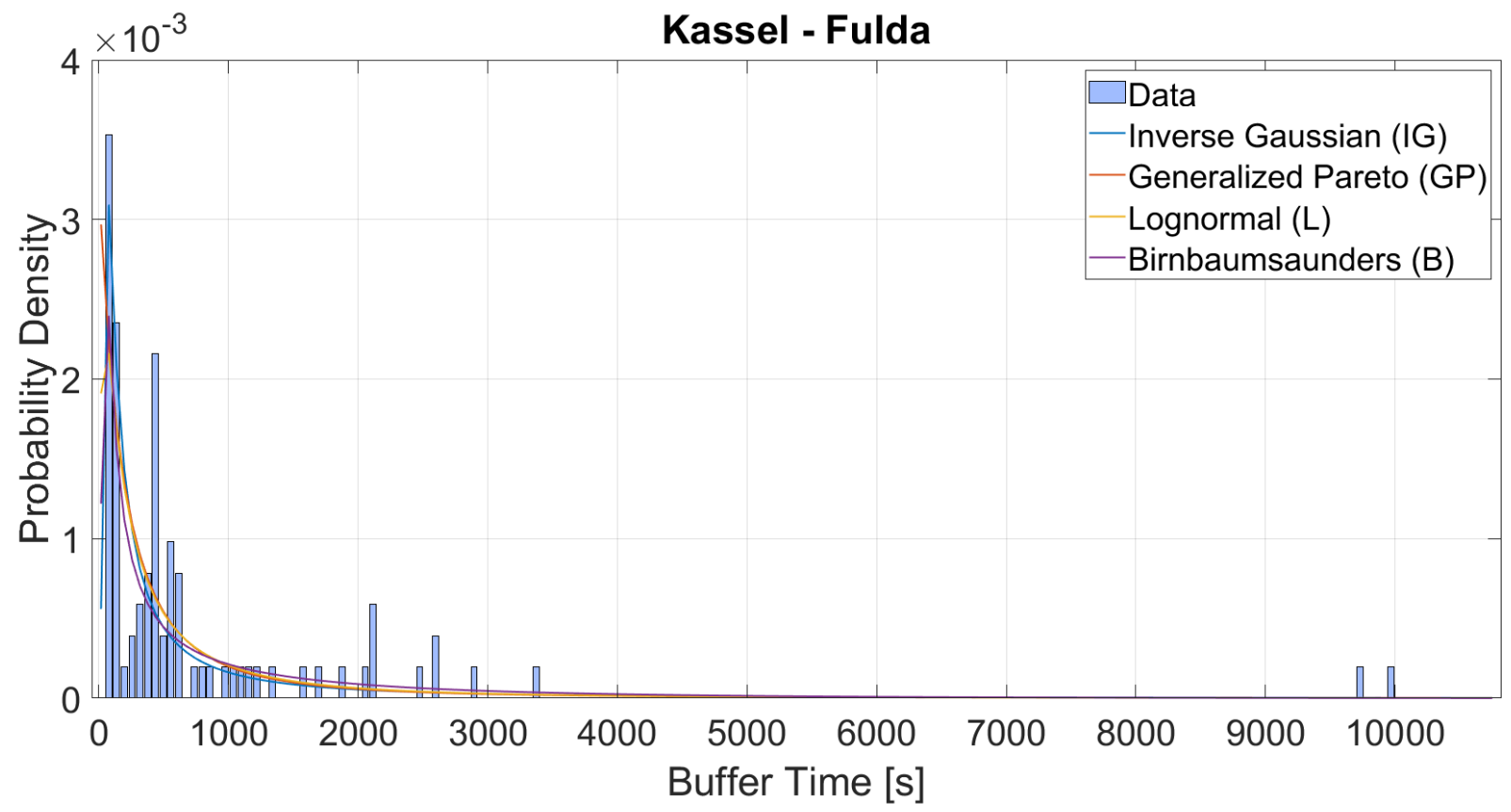

Figure 3: Probability density functions of best fitting buffer time distributions

parametric probability distributions are depicted. Additionally, the differences of the AIC-values to the best fitting distribution as well as the $p$-values according to the KS-test are given in Table 2. The suspected buffer time distribution is tested against the data set and rejected in case of $p<0.05$. 


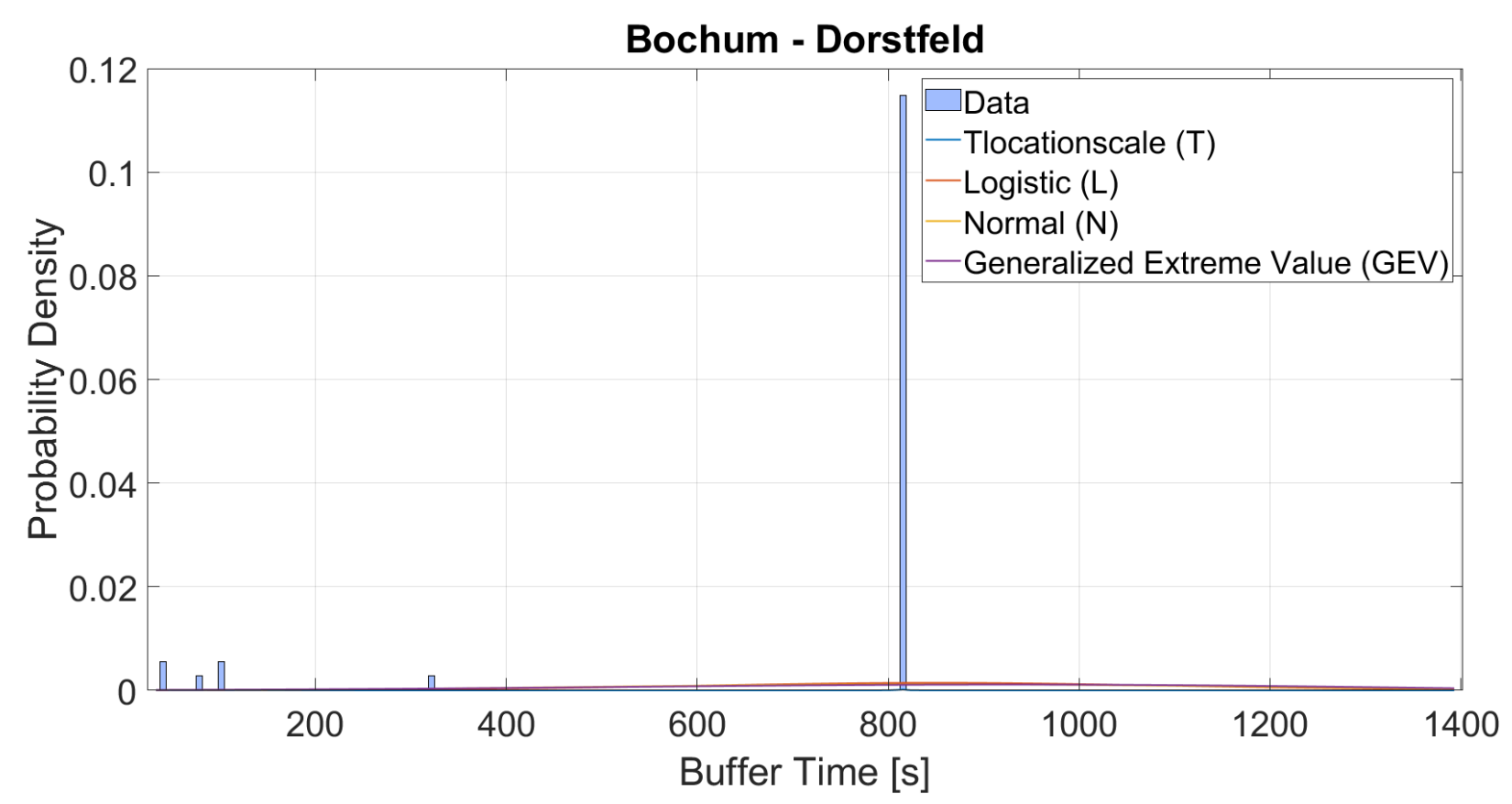

Figure 4: Probability density functions of best fitting buffer time distributions

It can be seen, that depending on the structure of the schedule, various distributions may be considered. Especially for highly cyclic schedules, e.g. for the suburban railway line Bochum-Dorstfeld where commuter trains are operated exclusively, it is possible that there is no good-fitting parametric probability distribution at all. In Figure 4 every parametric continuous distribution has to be rejected according to the KS-test. Hence, the only valid option may be to use the Degenerate distribution to map the actual buffer times from the schedule to a model.

\subsection{Influence of Buffer Time Distributions}

For the influence of buffer time distributions we consider two scenarios. The first one is the generic example used in section 3.1, above. Exponential distributions, Normal distributions with variation $\sigma^{2}=1$, Erlang distributions with shape parameter $k=3$, as well as Degenerate distribution are examined. All distributions are scaled to the same mean buffer time, such that, on average, the buffer times are equivalent for every pair of trains.

\section{Scenario 1}

In Figure 5 the expected knock-on delays per train for the above mentioned distributions are depicted over the mean buffer time. Additionally the current Level of Service (LoS) in German Railways DB Netz AG (2008) is added to demonstrate the differences in the evaluation and can be understood as the expected knock-on delay $E T_{k n o c k-o n}$ per train that results in an optimal quality. Hence, the point of intersection between expected knock-on delays and accepted height of knock-on delays by the rulebook is of special interest. To obtain the limit, train dispatchers were interviewed on the quality of flow on their surveyed railway line. 


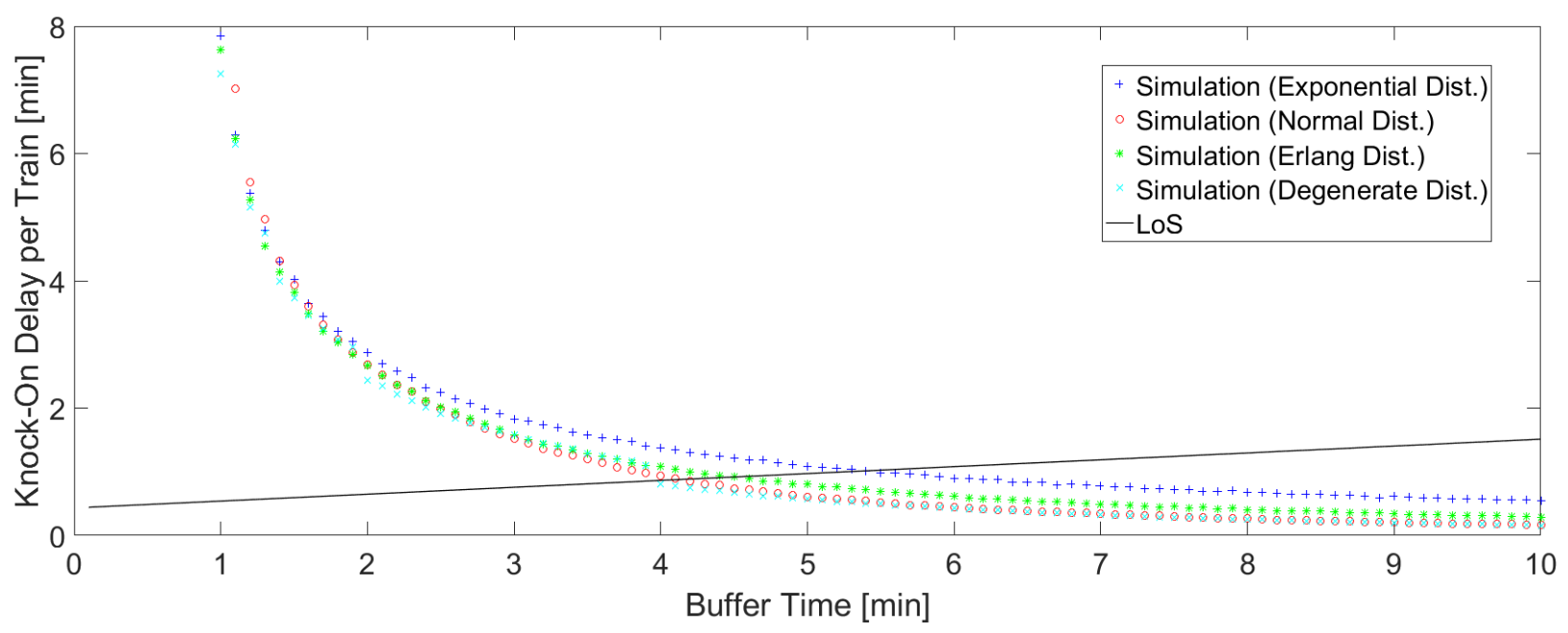

Figure 5: Influence of buffer time distributions - Scenario 1

The LoS is calculated by the following equation, which has been derived in Schwanhäußer and Schultze (1982) and is the current standard specified in Directive 405 of DB Netz AG (2008).

$L o S=0.257 \cdot \exp \left(-1.3 \cdot p_{p t}\right) \cdot t_{s c h e d}$

with

$p_{p t}$ - percentage of passenger trains,

$t_{\text {sched }}$ - length of the schedule.

The length of the schedules is in fact distinct for the considered distributions. Since the relative error between mean size of schedules and the original schedules of the distributions is on average less than $1.5 \%$ the error made by the usage of the average schedule length seems negligible.

With the knowledge of the minimum buffer time $t_{b, \min }$ - the point of intersection between expected waiting times and Level of Service - it is additionally possible to calculate the nominal capacity $N C$ for an arbitrary observed time horizon $t_{h}$ :

$$
N C=\frac{t_{h}}{t_{b, \min }+\bar{h}}
$$

All chosen distributions follow the course of declining mean waiting times with increasing mean buffer time. In contrast to the other chosen distributions the Exponential distribution does not concentrate its probability density in a close range. Therefore, it is more likely that extreme values occur.

The points of intersection for Degenerate and Normal distribution are located around minute 4, respectively 4.1, for Erlang distribution around minute 4.5 and for Exponential distribution at minute 5.4. These minimum buffer times correspond to 37 (Degenerate and Normal distribution), 35 (Erlang(3) distribution) respectively 31 trains (Exponential distribution) which can be operated in a 5-hour time-frame at the same quality.

For practical capacity issues this means that for schedules that are constructed with the considered buffer time distributions a difference of around 80 seconds per train may be necessary to retain a reasonable 
quality standard. Second, the analysis clearly shows that it is important to take the generated buffer time distribution into account. When constructing a schedule, different buffer time distributions lead to significantly differing knock-on delays and as a result to different capacity values. This is particularly relevant when both highly structured cyclic traffic and random mixed traffic services are to be compared.

\section{Scenario 2}

In Figure 6, a second instance for the influence of buffer time distributions is given. The input data, which is given in Appendix B, corresponds to a more heterogeneous train program. The point of intersection for all distributions, apart from Exponential, is approximately minute 7.8 - corresponding to 21 trains in 5 hours - whereas the Exponential distribution intersects the $L o S$ at minute 9 - corresponding to 19 trains in 5 hours. Thence, to gain the same Level of Service lines with exponentially distributed schedules require circa 70 seconds additional buffer time per train.

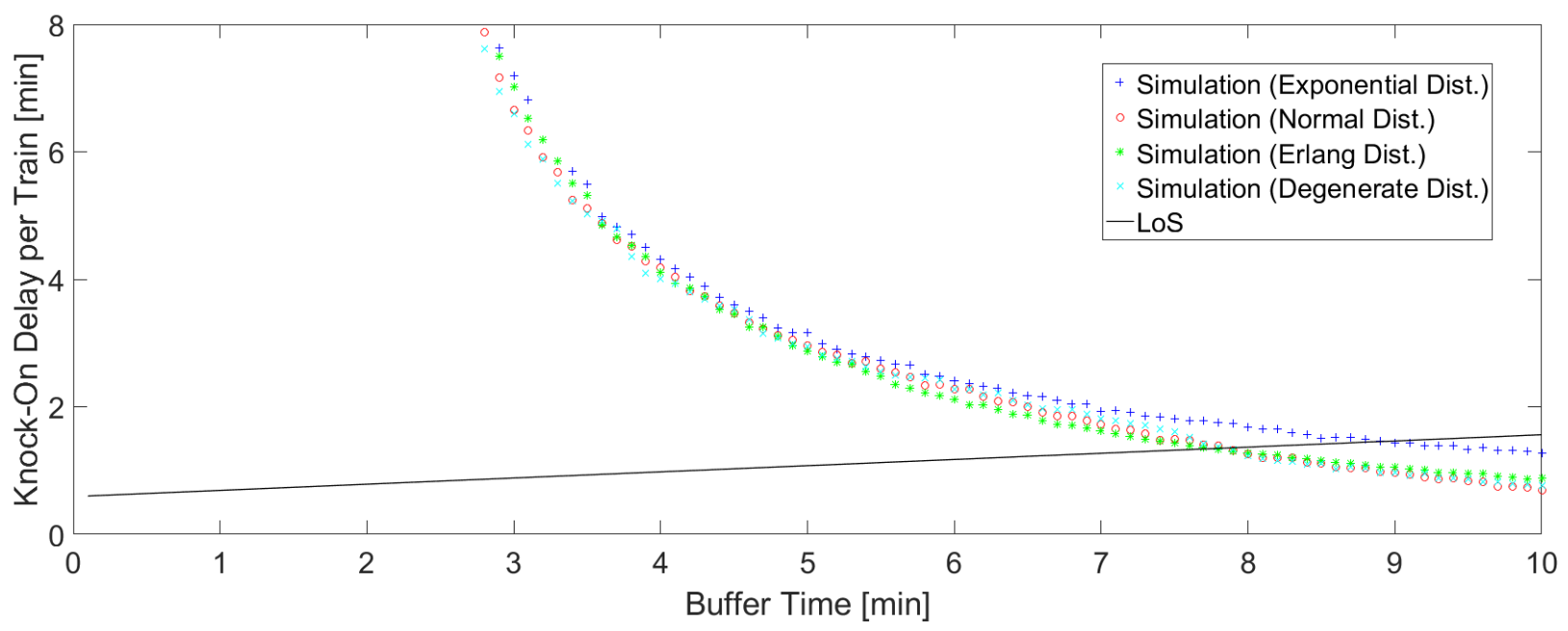

Figure 6: Influence of buffer time distributions - Scenario 2

\subsection{Sensitivity Analysis}

In the following section the influence of parameters on the point of intersection with the $L o S$ regarding the observed buffer time distributions is examined. The reference scenario consists of 6 train types (two long-distance model trains, two local model trains, two freight model trains) as follows:

- minimum headway time $h=5$ minutes for all trains

- average probability of delay $p_{d e l}=(0.5,0.5,0.4,0.4,0.6,0.6)$

- average amount of delay for the delayed trains $t_{\text {del }}=(5,5,2,2,20,20)$

- train mix $(0.1,0.2,0.2,0.2,0.2,0.1)$

For the simulation 300 trains and 500 runs are considered. Buffer times are incremented in steps of 0.1 minutes. It yields the following points of intersection $t_{b, \min }$ with the $L o S$ as measurement of acceptable operation quality and corresponding nominal capacity $N C$ in a 5-hour time-frame: 
- Exponential Dist.: $t_{b, \min }=7.0, N C=25.0$

- Normal Dist.: $t_{b, \min }=5.5, N C=28.6$

- $\operatorname{Erlang}(3)$ Dist.: $t_{b, \min }=6.1, N C=27.0$

- Degenerate Dist.: $t_{b, \min }=5.1, N C=29.7$

- STRELE: $t_{b, \min }=6.9, N C=25.2$

In the following, the sensitivity of the reference scenario towards variations of minimum headway time, average probability of delay and average amount of delay of the delayed trains is observed, respectively. The results are noted as $t_{b, \min } / N C$.

\begin{tabular}{|c|c|c|c|c|c|}
\hline$h$ & $\begin{array}{c}\text { Exponential } \\
\text { Dist. }\end{array}$ & $\begin{array}{c}\text { Normal } \\
\text { Dist. }\end{array}$ & $\begin{array}{c}\text { Erlang(3) } \\
\text { Dist. }\end{array}$ & $\begin{array}{c}\text { Degenerate } \\
\text { Dist. }\end{array}$ & STRELE \\
\hline \hline 1 & $1.7 / 44.8$ & $1.5 / 46.2$ & $1.6 / 45.5$ & $1.4 / 46.9$ & $1.6 / 45.5$ \\
\hline 2 & $3.3 / 36.1$ & $2.7 / 39.0$ & $2.9 / 38.0$ & $2.6 / 39.5$ & $3.2 / 36.6$ \\
\hline 3 & $4.5 / 31.6$ & $3.7 / 34.5$ & $4.0 / 33.3$ & $3.5 / 35.3$ & $4.6 / 31.3$ \\
\hline 4 & $5.7 / 28.0$ & $4.7 / 31.0$ & $5.0 / 30.0$ & $4.2 / 32.6$ & $5.8 / 27.8$ \\
\hline 5 & $7.0 / 25.0$ & $5.5 / 28.6$ & $6.1 / 27.0$ & $5.1 / 29.7$ & $6.9 / 25.2$ \\
\hline 6 & $8.0 / 23.0$ & $6.4 / 26.3$ & $7.0 / 25.0$ & $6.1 / 27.0$ & $7.9 / 23.2$ \\
\hline 7 & $9.0 / 21.4$ & $7.2 / 24.6$ & $7.8 / 23.4$ & $7.0 / 25.0$ & $8.8 / 21.7$ \\
\hline 8 & $10.1 / 19.9$ & $8.1 / 22.9$ & $8.9 / 21.6$ & $8.0 / 23.0$ & $9.5 / 20.6$ \\
\hline 9 & $11.1 / 18.6$ & $8.9 / 21.6$ & $9.5 / 20.7$ & $9.0 / 21.4$ & $10.2 / 19.7$ \\
\hline 10 & $12.0 / 17.6$ & $9.8 / 20.3$ & $10.4 / 19.5$ & $9.9 / 20.1$ & $10.9 / 18.9$ \\
\hline 11 & $13.0 / 16.7$ & $10.6 / 19.2$ & $11.0 / 18.8$ & $10.9 / 18.9$ & $11.5 / 18.1$ \\
\hline 12 & $13.8 / 16.0$ & $11.6 / 18.1$ & $11.9 / 17.8$ & $11.9 / 17.8$ & $12.0 / 17.6$ \\
\hline
\end{tabular}

Table 3: Point of intersection $t_{b, \min }$ with $\operatorname{LoS}$ and nominal capacity $N C$ (no. of trains) for various buffer time distributions and STRELE for different minimum headway times $h$

In Table 3 the impact of deviations in the amount of minimum headway times is depicted for various buffer time distributions and the STRELE-formula. The nominal capacity of the exponentially distributed scenario follows the course of the STRELE formula, but it seems noteworthy that the STRELE-formula seems to overestimate the nominal capacity due to averaging the delay characteristics. Additionally, it can be observed that highly cyclic schedules seem to result in higher nominal capacities. The difference in this particular scenario to STRELE in terms of nominal capacity is nearly 1 train per hour for minimum headway times of 4 minutes.

Table 4 shows the influence of varying delay probabilities as input parameter for the given scenario. It can be seen that, in general, simulations with exponentially distributed buffer times produce results similar to the STRELE-formula. The nominal capacity for the other observed buffer times distributions tends to be higher. It seems particularly notable that the effects on the nominal capacity diminish slowly with increasing 


\begin{tabular}{|c|c|c|c|c|c|}
\hline$p_{d e l}$ & $\begin{array}{c}\text { Exponential } \\
\text { Dist. }\end{array}$ & $\begin{array}{c}\text { Normal } \\
\text { Dist. }\end{array}$ & $\begin{array}{c}\text { Erlang(3) } \\
\text { Dist. }\end{array}$ & $\begin{array}{c}\text { Degenerate } \\
\text { Dist. }\end{array}$ & STRELE \\
\hline \hline 0 & $0.0 / 60.0$ & $0.0 / 60.0$ & $0.0 / 60.0$ & $0.0 / 60.0$ & $0.0 / 60.0$ \\
\hline 0.1 & $3.2 / 36.6$ & $3.4 / 35.7$ & $3.3 / 36.1$ & $3.5 / 35.3$ & $2.6 / 39.5$ \\
\hline 0.2 & $4.5 / 31.6$ & $4.1 / 33.0$ & $4.0 / 33.3$ & $4.8 / 30.6$ & $4.2 / 32.6$ \\
\hline 0.3 & $5.3 / 29.1$ & $4.6 / 31.3$ & $4.7 / 30.9$ & $4.9 / 30.3$ & $5.4 / 28.8$ \\
\hline 0.4 & $6.0 / 27.3$ & $4.9 / 30.3$ & $5.3 / 29.1$ & $5.0 / 30.0$ & $6.3 / 26.6$ \\
\hline 0.5 & $6.7 / 25.6$ & $5.2 / 29.4$ & $5.7 / 28.0$ & $5.0 / 30.0$ & $7.0 / 25.0$ \\
\hline 0.6 & $7.2 / 24.6$ & $5.5 / 28.6$ & $6.0 / 27.3$ & $5.3 / 29.1$ & $7.5 / 24.0$ \\
\hline 0.7 & $7.6 / 23.8$ & $5.8 / 27.8$ & $6.4 / 26.3$ & $5.6 / 28.3$ & $7.9 / 23.3$ \\
\hline 0.8 & $7.9 / 23.3$ & $6.0 / 27.3$ & $6.7 / 25.6$ & $6.0 / 27.3$ & $8.2 / 22.7$ \\
\hline 0.9 & $8.1 / 22.9$ & $6.3 / 26.6$ & $7.0 / 25.0$ & $6.2 / 26.8$ & $8.4 / 22.4$ \\
\hline 1 & $8.3 / 22.6$ & $6.5 / 26.1$ & $7.1 / 24.8$ & $6.6 / 25.8$ & $8.4 / 22.4$ \\
\hline
\end{tabular}

Table 4: Point of intersection $t_{b, \text { min }}$ with $L o S$ and nominal capacity $N C$ (no. of trains) for various buffer time distributions and STRELE for different average probabilities of delay $p_{d e l}$

probability of delay since the arrival of the trains gets stronger randomised and hence closer to a queuing perspective.

\begin{tabular}{|c|c|c|c|c|c|}
\hline $\begin{array}{c}\text { factor multiplied } \\
\text { with } t_{\text {del }}\end{array}$ & $\begin{array}{c}\text { Exponential } \\
\text { Dist. }\end{array}$ & $\begin{array}{c}\text { Normal } \\
\text { Dist. }\end{array}$ & $\begin{array}{c}\text { Erlang(3) } \\
\text { Dist. }\end{array}$ & $\begin{array}{c}\text { Degenerate } \\
\text { Dist. }\end{array}$ & STRELE \\
\hline \hline 0.25 & $5.1 / 29.7$ & $4.3 / 32.3$ & $4.4 / 31.9$ & $4.6 / 31.3$ & $3.7 / 34.5$ \\
\hline 0.5 & $5.9 / 27.5$ & $4.8 / 30.6$ & $5.1 / 29.7$ & $4.9 / 30.3$ & $5.4 / 28.8$ \\
\hline 0.75 & $6.6 / 25.8$ & $5.2 / 29.4$ & $5.6 / 28.3$ & $5.0 / 30.0$ & $6.3 / 26.5$ \\
\hline 1 & $7.0 / 25.0$ & $5.5 / 28.6$ & $5.9 / 27.5$ & $5.0 / 30.0$ & $6.9 / 25.2$ \\
\hline 1.25 & $7.3 / 24.4$ & $5.7 / 28.0$ & $6.4 / 26.3$ & $5.4 / 28.9$ & $7.3 / 24.4$ \\
\hline 1.5 & $7.5 / 24.0$ & $6.0 / 27.3$ & $6.5 / 26.1$ & $5.7 / 28.0$ & $7.6 / 23.8$ \\
\hline 1.75 & $7.8 / 23.4$ & $6.2 / 26.8$ & $6.7 / 25.6$ & $6.0 / 27.2$ & $7.8 / 23.4$ \\
\hline 2 & $7.9 / 23.3$ & $6.4 / 26.3$ & $7.1 / 24.8$ & $6.2 / 26.8$ & $8.0 / 23.1$ \\
\hline 2.25 & $8.0 / 23.1$ & $6.5 / 26.1$ & $7.3 / 24.4$ & $6.5 / 26.1$ & $8.1 / 22.9$ \\
\hline 2.5 & $8.3 / 22.6$ & $6.8 / 25.4$ & $7.3 / 24.4$ & $6.6 / 25.9$ & $8.2 / 22.7$ \\
\hline 2.75 & $8.2 / 22.7$ & $6.8 / 25.4$ & $7.5 / 24.0$ & $6.7 / 25.6$ & $8.3 / 22.6$ \\
\hline 3 & $8.5 / 22.2$ & $7.0 / 25.0$ & $7.5 / 24.0$ & $6.9 / 25.2$ & $8.4 / 22.4$ \\
\hline
\end{tabular}

Table 5: Point of intersection $t_{b, \min }$ with $L o S$ and nominal capacity $N C$ (no. of trains) for various buffer time distributions and STRELE for different average heights of delay of the delayed trains $t_{d e l}$

Table 5 demonstrates the effect of the amount of delay of the delayed trains on the minimum admissible 
average buffer time and the corresponding nominal capacity. As before, the samples with exponentially distributed buffer times stick closely to the STRELE-formula. The impact of variations of $t_{d e l}$ on the nominal capacity seem rather small. In the last case late freight trains arrive on average 60 minutes after their planned departure. Still, it was found that waiting times for these trains remain stable.

It can be observed that the Exponential distribution is affected the most by changes in the parameters, produces the highest knock-on delays and therefore requires a high amount of buffer time to perform adequate in operations. Degenerate and Normal distribution seem to cope best with changing parameters and generate in general smaller knock-on delays and hence higher nominal capacities. It is additionally notable that by not only fitting data with Exponential distribution, but allowing extra phases towards an Erlang distribution, the mass of probability density is concentrated more closely and hence produces more stable schedules. We therefore conclude that it is advantageous to have centred buffer time distributions to achieve stable operations due to relatively equal distributed buffer times.

\section{Conclusion}

In the present paper, the importance of realistic buffer time distributions representing actual timetable characteristics in (stochastic) delay propagation modelling for capacity analysis is investigated. We showed that the choice of buffer time has a significant influence on the expected knock-on delays and thus capacity. Differences of up to 1 minute buffer time per train, on average, were found to be necessery to achieve the same level of service. Line capacity, was found to vary by as much as $17 \%$. As a result, an enhancement of delay propagation models in capacity analysis with respect to buffer time representation seems advisable.

Acknowledgements. This work was supported by the German Research Council (DFG) Research Training Group 2236 "UnRAVeL - Uncertainty and Randomness in Algorithms, Verification and Logic" and Research Grant 283085490 "Integral capacity and reliability analysis of guided transport systems based on analytical models" as well as by DB Netz AG with the project "IEBWU". 


\section{References}

M. Abril, F. Barber, L. Ingolotti, M.A. Salido, P. Tormos, and A. Lova. An assessment of railway capacity. Transportation Research Part E: Logistics and Transportation Review, 44(5):774 - 806, 2008. ISSN 1366-5545. URL http: //www.sciencedirect.com/science/article/pii/S1366554507000579.

B. Adenso-Diaz, M. O. González, and P. González-Torre. On-line timetable re-scheduling in regional train services. Transportation Research Part B: Methodological, 33(6):387-398, 1999.

H. Akaike. A new look at the statistical model identification. IEEE transactions on automatic control, 19(6):716-723, 1974.

E. V. Andersson, A. Peterson, and J. Krasemann Törnquist. Quantifying railway timetable robustness in critical points. Journal of Rail Transport Planning \& Management, 3(3):95-110, 2013.

T. Büker and B. Seybold. Stochastic modelling of delay propagation in large networks. Journal of Rail Transport Planning E Management, 2 (1):34-50, 2012.

R. L. Burdett and E. Kozan. Techniques for inserting additional trains into existing timetables. Transportation Research Part B: Methodological, 43(8-9):821-836, Sep 2009. ISSN 0191-2615.

V. Cacchiani and P. Toth. Nominal and robust train timetabling problems. European Journal of Operational Research, 219 (3):727-737, 2012. ISSN 0377-2217. doi: 10.1016/j.ejor.2011.11.003.

V. Cacchiani, F. Furini, and M. P. Kidd. Approaches to a real-world train timetabling problem in a railway node. Omega, 58: 97-110, Jan 2016. doi: 10.1016/j.omega.2015.04.006.

G. W Corder and D. I. Foreman. Nonparametric statistics: A step-by-step approach. John Wiley \& Sons, 2014.

F. Corman, A. D'Ariano, and I. A. Hansen. Evaluating disturbance robustness of railway schedules. Journal of Intelligent Transportation Systems, 18(1):106-120, 2014.

B. Cule, B. Goethals, S. Tassenoy, and S. Verboven. Mining train delays. In International Symposium on Intelligent Data Analysis, pages 113-124. Springer, 2011.

W. Daamen, R. M. P. Goverde, and I. A. Hansen. Non-discriminatory automatic registration of knock-on train delays. Networks and Spatial Economics, 9(1):47-61, 2009.

DB Netz AG. Richtlinie 405 "fahrwegkapazität". Stand 01.01, 2008.

X. Delorme, X. Gandibleux, and J. Rodriguez. Stability evaluation of a railway timetable at station level. European Journal of Operational Research, 195(3):780-790, 2009.

T. Dewilde, P. Sels, D. Cattrysse, and P. Vansteenwegen. Improving the robustness in railway station areas. European Journal of Operational Research, 235(1):276 - 286, 2014. ISSN 0377-2217. doi: https://doi.org/10.1016/j.ejor.2013.10.062. URL http://www.sciencedirect.com/science/article/pii/S0377221713008916.

I. Gast. Wartezeiten infolge stehender Überholungen auf Eisenbahnstrecken, volume 39. Institute of Transport Science, RWTH Aachen University, 1986.

R. M. P. Goverde and L. Meng. Advanced monitoring and management information of railway operations. Journal of Rail Transport Planning \& Management, 1(2):69-79, 2011.

T. Huisman, R. J. Boucherie, and N. M. van Dijk. A solvable queueing network model for railway networks and its validation and applications for the netherlands. European Journal of Operational Research, 142(1):30-51, 2002. doi: 10.1016/S0377-2217(01)00269-7.

D. Janecek and F. Weymann. LUKS - Analysis of lines and junctions. In Proceedings of the 12th World Conference on Transport Research (WCTR), 2010.

P. Jovanović, P. Kecman, N. Bojović, and D Mandić. Optimal allocation of buffer times to increase train schedule robustness. European Journal of Operational Research, 256(1):44-54, 2017.

F. Khoshniyat and A. Peterson. Improving train service reliability by applying an effective timetable robustness strategy. Journal of Intelligent Transportation Systems, 21(6):525-543, Jun 2017. ISSN 1547-2450. doi: 10.1080/15472450.2017. 1326114

F. Khoshniyat and J. Törnquist Krasemann. An optimization approach for on-demand railway slot allocation. In 7th International Conference on Railway Operations Modelling and Analysis, RailLille2017, page 116447, 2017.

F. A. A. Kingdom and N. Prins. Chapter 9 - model comparisons. In F. A.A. Kingdom and N. Prins, editors, Psychophysics (Second Edition), pages 247 - 307. Academic Press, San Diego, second edition edition, 2016. ISBN 978-0-12-407156-8. doi: https://doi.org/10.1016/B978-0-12-407156-8.00009-8. URL https://www.sciencedirect.com/science/article/pii/ B9780124071568000098. 
R. M. Lusby, J. Larsen, and S. Bull. A survey on robustness in railway planning. European Journal of Operational Research, 2017.

L. E. Meester and S. Muns. Stochastic delay propagation in railway networks and phase-type distributions. Transportation Research Part B: Methodological, 41(2):218-230, Feb 2007. ISSN 0191-2615. doi: 10.1016/j.trb.2006.02.007.

L. Mussone and R. Wolfler Calvo. An analytical approach to calculate the capacity of a railway system. European Journal of Operational Research, 228(1):11-23, 2013. doi: 10.1016/j.ejor.2012.12.027.

L. Nie and I. Hansen. System analysis of train operations and track occupancy at railway stations. European Journal of Transport and Infrastructure Research, 5(1):31-54, 2005.

N. Nießen. Leistungskenngrößen für Gesamtfahrstraßenknoten. PhD Thesis, RWTH Aachen University, 2008. URL http: //publications.rwth-aachen.de/record/687127.

M. Salido, F. Barber, and L. Ingolotti. Robustness in railway transportation scheduling. In Intelligent Control and Automation, 2008. WCICA 2008. 7th World Congress on, pages 2880-2885. IEEE, 2008.

W. Schwanhäußer. Die Bemessung der Pufferzeiten im Fahrplangefüge der Eisenbahn. PhD thesis, Verkehrswissenschaftliches Institut der RWTH Aachen, 1974.

W. Schwanhäußer. The status of german railway operations management in research and practice. Transportation Research Part A: Policy and Practice, 28(6):495-500, 1994.

W. Schwanhäußer and K. Schultze. Ermittlung von Qualitätsmaßstäben für die Berechnung der Leistungsfähigkeit eines Streckenabschnittes und Entwicklung eines Rechenverfahrens zur Ermittlung von Endverspätungen: Forschungsarbeit für die Deutsche Bundesbahn. na, 1982.

W. Schwanhäußer, I. Gast, K. Schultze, H. Wakob, O. Brünger, and S. Mehta. Programmfamilie sls-stresi. STRELE, ALFAPC-Programme zur Leistungsfähigkeitsberechnung und Simulation Benutzerhandbuch, Version, 4, 2000.

M. Sheppard. Allfitdist [fit all valid parametric probability distributions to data], 2012.

E. Solinen, G. Nicholson, and A. Peterson. A microscopic evaluation of railway timetable robustness and critical points. Journal of Rail Transport Planning \&S Management, 7(4):207 - 223, 2017. ISSN 2210-9706. doi: https://doi.org/10.1016/j.jrtpm. 2017.08.005. URL http://www.sciencedirect.com/science/article/pii/S2210970617300392.

Y. Takeuchi, N. Tomii, and C. Hirai. Evaluation method of robustness for train schedules. Quarterly Report of RTRI, 48(4): 197-201, 2007.

UIC. Code 406 - Capacity, 1st edition. Tech. Report, International Union of Railways, 2004.

UIC. Code 406 - Capacity, 2nd edition. Tech. Report, International Union of Railways, 2013.

N. Weik and N. Nießen. A quasi-birth-and-death process approach for integrated capacity and reliability modeling of railway systems. Journal of Rail Transport Planning \& Management, 7(3):114-126, Dec 2017. doi: 10.1016/j.jrtpm.2017.06.001.

N. Weik, N. Niebel, and N. Nießen. Capacity analysis of railway lines in germany-a rigorous discussion of the queueing based approach. Journal of Rail Transport Planning \& Management, 6(2):99-115, 2016.

C. Wen, Z. Li, Y. Tang, H. Ping, Y. Yang, and J. Lessan. Statistics of hsr primary delay based on real-world records: Case of wuhan-guangzhou. In 7th International Conference on Railway Operations Modelling and Analysis, RailLille2017, page 118367, 2017.

E. Wendler. The scheduled waiting time on railway lines. Transportation Research Part B: Methodological, $41(2): 148$ 158, 2007. ISSN 0191-2615. URL http://www.sciencedirect.com/science/article/pii/S019126150600018X. Advanced Modelling of Train Operations in Stations and Networks.

E. Wendler, N. Nießen, and E. Schröter. Empirical analysis of capacity consumption for the new capacity leaflet of uic. Gutachten im Auftrag des Internationalen Eisenbahnverbandes UIC, Aachen, 2002.

J. Yuan. Stochastic Modelling of Train Delays and Delay Propagation in Stations. PhD thesis, Delft University of Technology, 2006.

J. Yuan and I. A. Hansen. Optimizing capacity utilization of stations by estimating knock-on train delays. Transportation Research Part B: Methodological, 41(2):202-217, 2007. 


\section{Appendix A. Pseudo-Code of the Simulation Environment}

Data: vector of arbitrarily distributed buffer times $t_{b}$ of size $n$, matrix of minimum headway times $h$ of size $c \times c$, vector of train ranks $r$ of size $n+1$, vector of probabilities of delay $p_{\text {del }}$ of size $n+1$, vector of average time of delay of the delayed trains $t_{d e l}$ of size $n+1$

Result: sum of knock-on delays $s_{k o d}$, schedule length $s_{l e n}$

// Schedule Generation (c.f. 2.2.1)

1 initialise matrix $s_{\text {sch }}$ of size $(n+1) \times 3$;

// First column for storing the scheduled departure time, initially set to 0

// Second column for storing the train number, intitially set to $1 . . n+1$

// Third column for storing the train rank $r$

2 for $i=2 . . n+1$ do

$3 \mid s_{s c h}(i, 1)=s_{s c h}(i-1,1)+t_{b}(i-1)+h(r(i-1), r(i))$;

// Delay Implementation (c.f. 2.2.2)

4 Initialise matrix $s_{o p}=s_{s c h}$;

$\mathbf{5}$ for $i=1 . . n+1$ do

$6 \quad$ if rand $\leq p_{\text {del }}(r(i))$ then

$7 \quad$ delay $=\operatorname{exprnd}\left(t_{\text {del }}(r(i))\right)$;

$8 \quad s_{o p}(i, 1)=s_{o p}(i, 1)+$ delay

// Rescheduling (c.f. 2.2.3)

9 Initialise $s_{\text {help }}=\operatorname{sortrows}\left(s_{o p},[3,1]\right)$;

// Sort the disturbed schedule by rank and actual departure time

10 Initialise $s_{\text {res }}(1,:)=s_{\text {help }}(1,:)$;

11 for $i=2 . . n+1$ do

12 if $s_{\text {help }}(i, 1)<s_{\text {res }}(1,1)$ then

$13 \mid$ if $s_{\text {help }}(i, 1)+h\left(s_{\text {help }}(i, 3), s_{\text {res }}(1,3)\right)<s_{\text {res }}(1,1)$ then

$14 \quad$ Sort $s_{\text {help }}(i, 1)$ in the free spot in front;

$15 \quad$ else

$16 \quad$ Sort $s_{\text {help }}(i, 1)$ in the next free spot in the middle;

$17 \quad$ Sort $s_{\text {res }}$ by departure time;

$18 \quad$ else if $s_{\text {help }}(i, 1) \geq s_{\text {res }}(1,1) \& s_{\text {help }}(i, 1)<s_{\text {res }}($ end,, 1$)$ then

$19 \quad$ Sort $s_{\text {help }}(i, 1)$ in the next free spot in the middle;

$20 \quad$ Sort $s_{\text {res }}$ by departure time;

$21 \quad$ else

$22 \quad$ Sort $s_{\text {help }}(i, 1)$ in the next free spot at the end;

$23 \quad$ Update $s_{\text {res }}$ by departure time;

// Evaluation of a Simulation Run (c.f. 2.2.4)

24 Calculate sum of knock-on delays $s_{k o d}=\sum_{i=1}^{n+1}\left(s_{o p}(i, 1)-s_{s c h}(i, 1)\right)$;

25 Set $s_{l e n}=s_{o p}(e n d, 1)$;

Algorithm 1: Algorithm sketch for the calculation of knock-on delays and schedule length in the proposed simulation tool 


\section{Appendix B. Input Parameters for Scenario 2 in Section 3.4}

In the second example we simulated $n=300$ trains with 500 runs in 0.1 minute steps. In Table B. 1 the minimum headway times for the second example are given for long distance trains (ldt), local trains (lt) and freight trains (ft). Table B.2 gives the remaining train parameters for the simulation.

\begin{tabular}{|c|c|c|c|c|c|c|}
\hline headway times & ldt1 & ldt2 & lt1 & lt2 & $\mathrm{ft} 1$ & $\mathrm{ft} 2$ \\
\hline ldt1 & 3 & 3 & 3 & 3 & 3 & 3 \\
\hline ldt2 & 4.4 & 4 & 4 & 4 & 4 & 4 \\
\hline lt1 & 6 & 5.5 & 5 & 5 & 5 & 5 \\
\hline lt2 & 8 & 7.5 & 6.2 & 6.2 & 5.8 & 5.8 \\
\hline $\mathrm{ft} 1$ & 10 & 10 & 8 & 8 & 7.6 & 7.6 \\
\hline $\mathrm{ft} 2$ & 13 & 13 & 12 & 11 & 11 & 9.3 \\
\hline
\end{tabular}

Table B.1: Minimum headway times for the second example

\begin{tabular}{|c|c|c|c|c|c|c|}
\hline & ldt1 & ldt2 & lt1 & lt2 & $\mathrm{ft} 1$ & $\mathrm{ft} 2$ \\
\hline$p_{\text {del }}$ & 0.5 & 0.5 & 0.6 & 0.25 & 0.6 & 0.6 \\
\hline$t_{\text {del }}$ & 5 & 5 & 4.5 & 2 & 10 & 10 \\
\hline probability in \% & 12.5 & 12.5 & 25 & 25 & 20 & 5 \\
\hline
\end{tabular}

Table B.2: Train parameters for the second example 